\title{
Tips for Building a Community for Incoming Medical Students Virtually in the COVID-19 Era
}

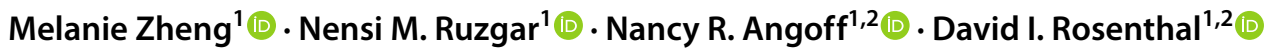

Accepted: 15 October 2021 / Published online: 4 November 2021

(c) The Author(s) under exclusive licence to International Association of Medical Science Educators 2021

\begin{abstract}
A sense of community benefits medical trainees by preserving mental well-being, nurturing collegiality and mentorship, and grounding ties with partnering organizations and services. Within medical school, building these support relationships often begins shortly after matriculation. In the current pandemic and the accompanying shift to a virtual class format, we believe that a dedicated effort to foster this sense of community is crucial for students who otherwise may feel untethered to their new learning environment. Here, we detail tips for building a medical school community virtually in the COVID-19 era between peers, within the school institution, and within the surrounding environment.
\end{abstract}

Keywords Course design $\cdot$ Student wellness $\cdot$ E-learning $\cdot$ Online learning $\cdot$ Mental health

\section{Introduction}

The ongoing COVID-19 pandemic has strained the healthcare workforce, leading to upended medical team structures and uncertainties over the capacity for medical training in this stressful time when providers are already over-stretched. Nevertheless, medical education has persisted and must continue to do so, as trained medical professionals are needed now more than ever. In 2019, over 21,000 students matriculated to medical school in the United States [1], and while there has been no published data for the 2020-2021 academic year at the time of this writing, the size of the corresponding incoming class at our own institution did not change. However, current and evolving guidelines over in-person meetings and restrictions to hospital visitation have affected how preclinical curriculum is traditionally delivered. Lectures and discussion sections may need to be in online or mixed formats, presenting its own learning curve. One often overlooked facet of a virtual introduction to medical school is the loss of in-person social interactions for new classmates. For a field that is increasingly concerned with burnout in its workforce and trainees and depends on the collaboration of health professionals, building a sense of

Melanie Zheng and Nensi Ruzgar equally contributed to this work.

Melanie Zheng

melanie.zheng@yale.edu

1 Yale University School of Medicine, New Haven, CT, USA

2 Yale School of Medicine, Section on General Internal Medicine, New Haven, CT, USA community for students is vital to promote resilience and prevent burnout [2,3].

Medical students express doubt and emotional hardening as early as their first year, making the facilitation of support systems early on in medical school imperative for their wellbeing and success [4]. To address this gap at our institution, a dedicated 2-week introductory course was developed in 2015 and has played a part in building a community among peers and introducing students to their future profession. In its earlier, pre-pandemic iterations, the course centered around not only orienting students to institution-specific resources (i.e., simulation center and main teaching hospital) with small groups to facilitate navigating these spaces, but also exploring themes in clinical care (e.g., cultural humility with a medical anthropology book club in 2015 and stacked lectures on the biopsychosocial facets of the U.S. opioid epidemic in 2017) that prompted group discussion. While different curricular activities could be transitioned to a virtual setting with varying degrees of ease and physical orientation activities were postponed, there was a concern that the introductory course would not be able to contribute to the goal of building a medical school community in the same fashion or that these sessions may be lost in translation without purposeful updating for a new, virtual format. Here, we detail the successes and challenges observed while implementing this curriculum for the first time in the virtual setting and summarize our takeaways into 10 tips for building a medical school community virtually in the COVID-19 era. Given that components of virtual curricula may remain for the foreseeable future depending on evolving infection 
protection provisions, each tip discusses sustainable virtual community building activities that can be employed in future years. Furthermore, each tips' purposeful consideration of community and inclusivity has utility beyond virtual limitations and can be beneficial in in-person or mixed formats of learning as well. As one's community can encompass different aspects of a medical student's experience, we have delineated three nested spheres: (1) classmate peers, (2) the broader campus environment, and, broader still, (3) the surrounding city environment (Fig. 1).

\section{Forming Community Among Peers}

\section{Tip 1: Facilitate Group Norm Formation}

In a learning environment with new acquaintances, it is helpful to first establish a list of commonly decided-upon ground rules and expectations [5]. Studies have found that lists of group norms serve future discussions more meaningfully when generated by students rather than course leaders [5,6]. Prior to our two week-curriculum, students were invited to a pre-curriculum session with an icebreaker followed by small group introductions. The Zoom setting offered unique advantages in the capacity to create breakout rooms to facilitate introductory discussions and to combine rooms to allow for nested discussions. Within the small groups led by upper-year students, firstyear students were tasked with generating a list of group norms for the class as a whole. Collaborating on a list of guidelines allowed students to arrive at a mutually agreed upon code of conduct so that students had actionable expectations for future discussions, especially in the case of disagreements.

In preparation, facilitators generated a list of sample group norms to seed the discussion but were encouraged to suggest them only if discussion had stalled. While this mitigation strategy was successful, another challenge we faced was creating an environment where students may not have felt comfortable disagreeing with a suggestion shortly after meeting their classmates, predisposing to groupthink. This was acknowledged in the event introduction to help encourage any opposing ideas but may remain a limitation to the activity. After all groups were satisfied with their generated list, group norms were collated by student course directors into common themes (Table 1).
Fig. 1 Proposed nested spheres of community for new students

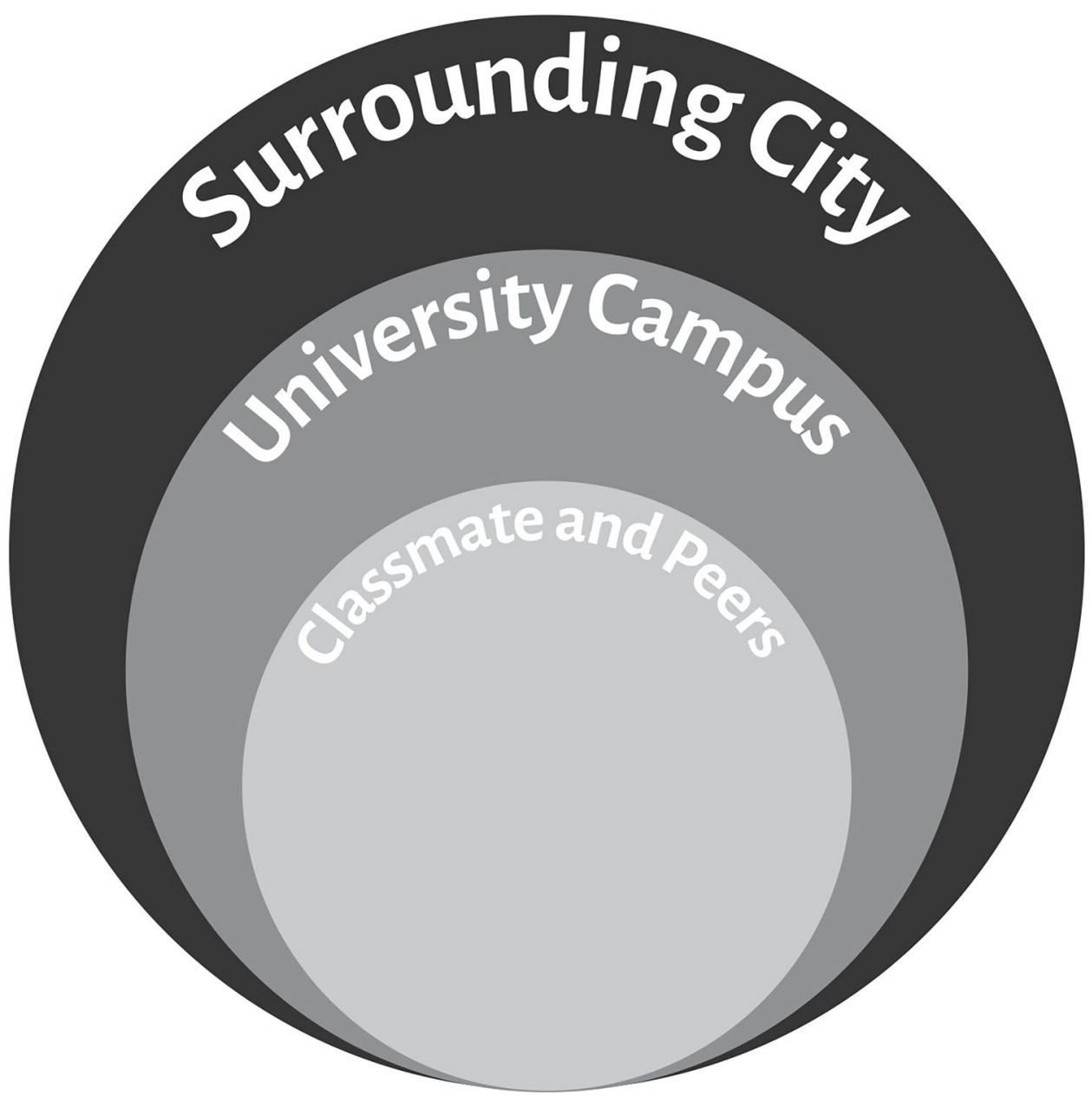


Table 1 Themes of student-generated group norms

\begin{tabular}{ll}
\hline & Theme \\
\hline 1 & Make space for everyone to participate \\
2 & Be present and engaged \\
3 & Keep conversations said in small groups confidential, \\
& do not repeat outside of sessions \\
4 & Speak with "I" and "me" statements \\
5 & Be respectful and graceful of everyone's opinions and \\
6 & ideas, even when we may disagree \\
7 & Be patient with technical difficulties \\
8 & Be considerate of others' time \\
\hline
\end{tabular}

Once organized, the cohesive list was sent to course directors, faculty, and students to serve as a guide for the course. As the students would be using similar formats for the foreseeable future in their preclinical curriculum, the generated list could also serve as a guide beyond the introductory course period.

\section{Tip 2: Establish Small Groups to Bolster the Group as a Whole}

Virtual curricula often limit organic conversations and introductions between peers. In our experience, online meeting platforms are not designed to accommodate multiple conversations in a meeting due to audio and visual limitations and are more conducive to single-speaker presentations [7]. This contrasts with an in-person encounter in which new classmates typically get to know one another through casual introductions, which are vital to students becoming comfortable discussing and sharing opinions with one another.

Given this pressing need to foster productive conversations as a class, the class of 104 was divided into eight small groups of thirteen, with an upper-year medical student serving as group student leader (SL) and point person. Groups were paired into teams for an additional layer of support (Fig. 2). These small groups were encouraged to schedule extracurricular social time and develop a sense of community supported by their SLs. Therefore, these groups facilitated more opportunities for one-on-one interactions and gave students a home base where they may feel more comfortable posing questions or concerns to a smaller group. While we observed a potential challenge for students to get to know others outside of their assigned groups, we also organized additional optional recreational themed Zoom sessions (i.e., baking, hiking, interval exercise training, and movie night) to foster further bonding through common interests. These approaches in turn aided in students feeling more comfortable among their class.

\section{Tip 3: Cultivate a Supportive Group-Learning Environment by Specific Use of Group-Learning Skills}

As medicine grows to integrate more collaborative learning and practice environments, there is an increasing recognition of the student behaviors that may impact its efficacy [8]. While programs have previously harnessed this information to train medical educators with effective facilitation skills, we aimed to equip the students directly with knowledge of and skills for effective group forming, collaboration, and group-based learning using virtual tools. A workshop was developed focusing on five steps of group work as it relates to medical education (Fig. 3).

The workshop included an introduction by a faculty expert in communication sciences with specific emphasis on using virtual communication to engage in group activity, including various applications and presentation of visible body language compared to written communication. This was followed by breakout groups where students were encouraged to apply the group-based learning framework led by trained peer facilitators, and a classwide debrief focusing on the takeaways from breakout group exercises. The peer facilitators received training on each of the five steps and were designated to act as either a "hands-on" or "hands-off" facilitator to provide students with a diversity of group-learning experiences. Hands-on facilitators were encouraged to provide more guidance, while hands-off facilitators were encouraged to "act as a fly on the wall and audiovisual helper"; both facilitator types were instructed to maintain appropriate standards for
Fig. 2 Organization of the incoming medical student cohort into teams and small groups to facilitate peer introductions

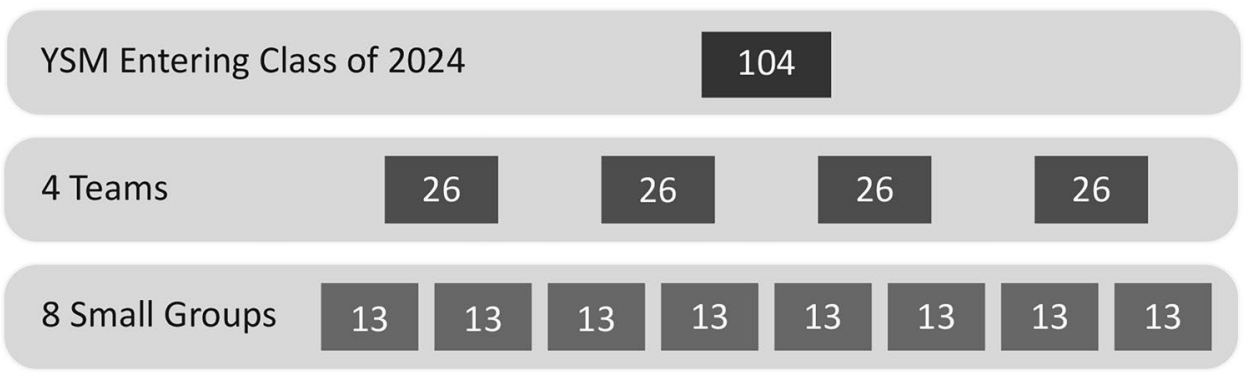




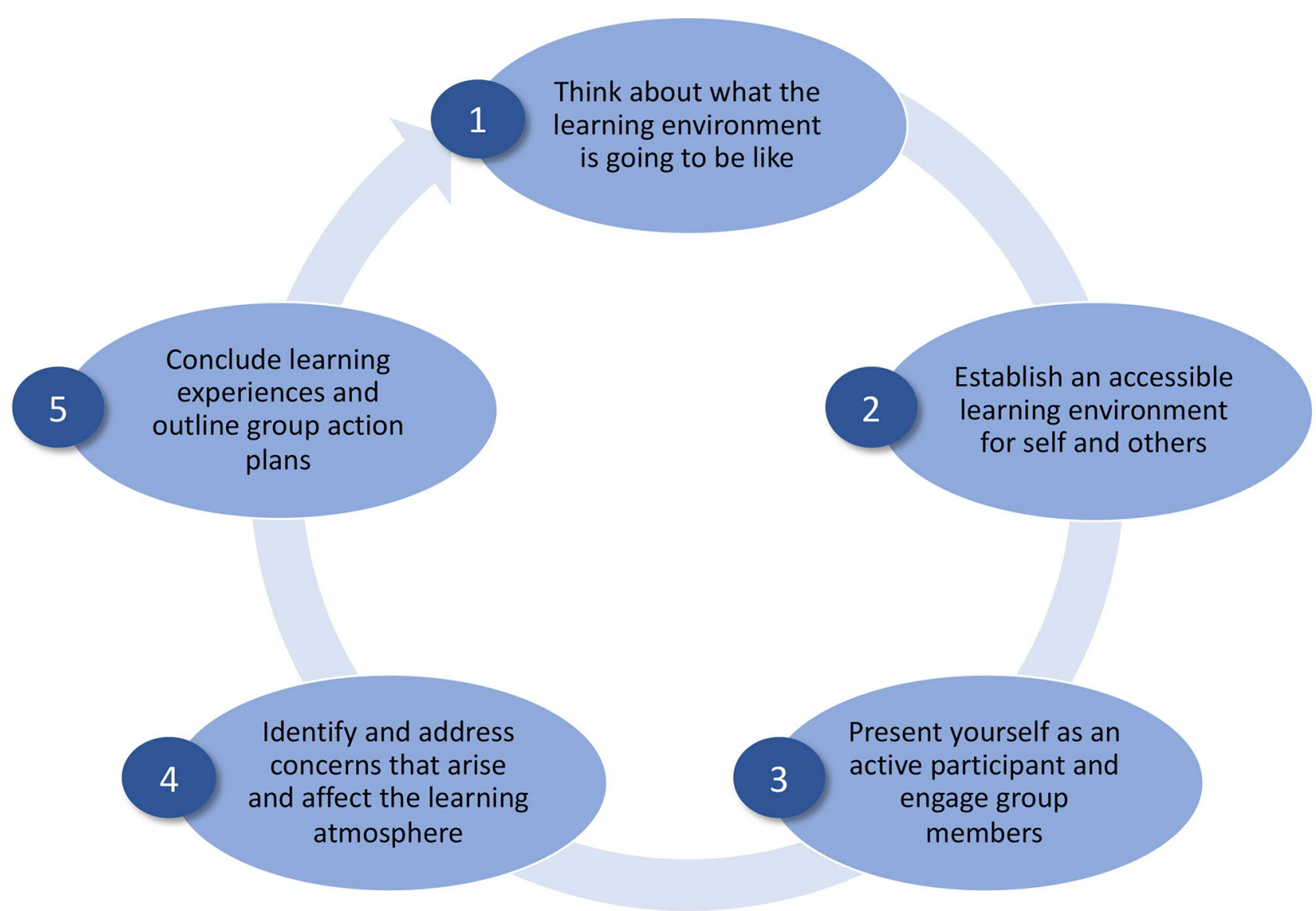

Fig. 3 The Five-Step Framework for Group-Based Learning

learning and intervene promptly if the learning environment became unsafe (e.g., discriminatory statements and unsupportive to other ideas in the brainstorming process) [9]. The debrief was intended to emphasize the opportunities to have conducive group experiences under either facilitator style, make students aware of the behaviors they implemented in a brief virtual exercise, and allow them to learn from each other's experiences.

Based on our experiences, some students in this activity were confused by the intentionally open-ended nature of the prompts (e.g., "pick a hobby as a group to encourage class cohesion"), which detracted from the overarching discussion of group-forming process. Future exercises may work to ensure that the breakout group exercises are either easily achievable, simple tasks that would leave enough time to discuss the group process (e.g., "deciding on a favorite book as a group") or relate directly to group formation.

\section{Tip 4: Create Environments for Reiterative Practice of Peer-to-Peer Constructive Feedback Giving}

Feedback giving can take many forms, including the more structured summative feedback model based traditionally on a finished product and the more dynamic exchange of formative feedback when peers brainstorm together [10]. Both scenarios build cohesion within a group, as it engages members in a conversation, incorporates students into each other's success, and establishes an interconnected achievement network.

Within our introductory course, feedback exchange was a key component of the culminating project in the form of the integrated hackathon, detailed further in Tip 8. Throughout stages of the process, students exchanged feedback within brainstorming discussions with one another, SLs, and community or faculty mentors. At the end of the challenge, each student also provided feedback on their peers' final projects based on a pre-set rubric (Supplementary Table 1). Providing a rubric with defined factors for evaluation in addition to asking students for feedback via free-text responses achieved a balance of guided peer evaluation and free exchange of ideas for improvement. Integrating these methods into a hackathon and into the larger introductory course empowered students to actively advocate for and benefit their peers' and individual learning. Because our students provided feedback on all final presentations, this led to additional time burden and subsequently some repetitive feedback 
as students tired from this process. We worked to allocate enough time for each presentation to be reviewed asynchronously in our curriculum. Similar future exercises may consider limiting the number of feedback-giving requirements for students based on their specific time constraints.

\section{Forming Community Within the Institution}

\section{Tip 5: Collate an Interdisciplinary Roster of Institutional Experts as Mentors and Teachers}

Beyond the students' introduction to their peers, an integral part of the medical school community depends on the support and guidance of the non-student members within the institution. In prior years, students would have the chance to meet faculty face-to-face through in-person didactics. Recognizing this, there was a concentrated effort to integrate more conversations with faculty and administrators into the curriculum.

In the virtual environment, an enriching way to integrate and introduce the experts at our institution was through their involvement in organizing and teaching parts of the introductory curriculum. Because the curriculum focused on the medical profession and the health disparities exacerbated by the COVID-19 pandemic, experts across a diverse range of fields were enlisted to lead lectures, panels, and small-group discussions. Each opportunity included question and answer periods as well as contact information for key stakeholders at the institution, allowing students to expand their institutional network further.

An interactive way to foster mentorship connections with experts at our institution is afforded through hackathons [11, 12] and was integrated into our introductory curriculum as well. Experts from clinical medicine, hospital administration, business, law, social work, education, and community engagement were recruited to serve on interdisciplinary panels. However, participation unfortunately varied drastically with scheduling availability; potential mentors with active in-person requirements in the workforce especially in the COVID-19 period (i.e., community workers and nurses) were less available to support activities. While we mitigated the potential imbalance by designing mentoring opportunities that required as little as $30 \mathrm{~min}$ of virtual meeting time, future activities should account for this potential imbalance during their recruitment and potentially feature some offhour mentoring opportunities on the weekend or in the evenings. These panels served as an incubator for conversation about student ideas and emphasized a culture of collaboration. Although the panels were brief, lasting 15-30 min, students were encouraged to reach out to pursue projects with mentors, providing an opportunity for continued engagement and mentorship.

\section{Tip 6: Integrate Near-Peer Mentorship into Curriculum with Upper-Year Students}

For any new medical student, it is crucial to establish points of contact as they navigate a new learning environment. Upper-year students in particular are important resources for newly entering students, as their experiences are the most timely and helpful in navigating the same spaces [13]. This is especially important in virtual curricula, where students are not expected to be present in-person.

To maximize first-year student interaction with upperyear students, the course was designed to feature upper-year students in different roles. In addition to the paid SLs closely interacting with assigned teams in scheduled and ad hoc virtual meetings, several curricular sessions involved students volunteering as one-time small group facilitators, workshop leaders, discussants, or panelists. There was an emphasis on featuring different student perspectives across these sessions, so that first-year students could appreciate the diverse array of student initiatives, interests, and extracurricular activities. However, it was challenging to represent key student populations that had less flexible schedules, including clerkship students and those actively applying to residency. SLs who had completed their clerkships were therefore asked to speak about their prior experiences to address these gaps. Many students used these opportunities to reach out to and connect with several upper-year students with shared interests, continuing to benefit from near-peer mentorship beyond the course.

\section{Tip 7: Acknowledge the Unique Challenges Presented}

While the support of upper-year students and faculty is a valuable part of the institutional community of any new medical student, it is important to acknowledge that this cohort will experience unique disruptions to their learning that prior years of medical trainees did not face on this scale [14].

Our introductory curriculum included a panel titled "How to Be a Medical Student," which showcased the difference in preclinical activities and learning styles among senior medical students. The session aimed to give the first-year students examples of how they might choose to spend their first year at medical school as they become acclimated to the new environment. The panel featured two second-year students, one returning $\mathrm{MD} / \mathrm{PhD}$ student, two research-year students, one student pursuing the MBA portion of his MD/ MBA degree, and one recent graduate. Of note, only the second-year panelists could remark on how their preclinical time was impacted by the shift to a virtual format. Feedback for this session underscored the unforeseen disconnect between upper-year students' experiences and the feasibility of activities for first-year students. For instance, a few of 
upper-year students' fondest memories from first year (i.e., getting to know classmates over lunch and certain in-person events) were no longer feasible in the same way for the new class and caused unintended disappointment.

Medical schools have made strides in bridging these gaps in social interaction and in-person experiences in the past few months, but the progress should be more transparent so that first-year students do not feel alienated from their institution and that they cannot engage in the "traditional" medical school experience [14]. A teaching point would be to acknowledge the fundamental differences in medical school experience due to limitations brought on by the pandemic and to communicate with students, faculty, and administrators how the medical school is mitigating these differences. In subsequent years, this disconnect between upper-year and first-year students may not be as distinct, as the upper-year students will also have familiarity with virtual-predominant learning and will be able to share experiences from a more relatable position.

\section{Forming Community with the Surrounding City}

\section{Tip 8: Host an Interactive Hackathon for Students to Actively Engage with Community Residents and Stakeholders}

Hackathons are innovative events traditionally focused on technology design and development, and task participants with posing solutions centered around a theme in team-based collaborative settings $[15,16]$. As they are increasingly applied to the field of medicine, the emphasis of teamwork and collaboration in hackathons can also be harnessed to encourage community building among students around curricular themes [12, 16-18].

In our introductory curriculum, a hackathon ran concurrently with the didactic components of the course and centered around the same theme of health inequities exacerbated by the pandemic. Students were asked to pitch inequities that they would like to address in a class-wide virtual meeting and were provided with an opportunity to network with peers in breakout groups based on their pitches. The students used these breakout rooms to discuss ideas and work together to form feasible projects. Over the following 2 weeks, students worked on their projects outside of curricular hours, applied concepts taught in the curriculum while designing solutions to address inequities in the New Haven community. Students were encouraged to seek feedback from stakeholders from New Haven throughout the hackathon and were provided dedicated periods with community-based mentors with ties to our institution. These opportunities allowed students to build stronger bonds with peers, while also learning more about the needs of the New Haven community and connecting their understanding of the didactic material with how they fit into their new city as future medical providers.
As with many hackathons, our event was also limited in the timeframe provided to design and propose projects. Given the time constraint, most projects remained in proposal stages and the students benefited from brainstorming within and outside of mentorship sessions; however, future events may consider providing further follow-up opportunities for student groups to develop their ideas more tangibly and move to the implementation stages.

\section{Tip 9: Showcase and Connect with Existing Efforts in the Community}

Highlighting community leaders and community organizations both contextualizes the curricular goals within the New Haven community and provides a separate network of mentors for students in their new city. In our curriculum, institutional affiliates leading a range of services in the community were invited to coordinate and moderate panels featuring their collaborators from the New Haven community. These services included health coordination for veterans, housing support for homeless populations, and case coordination for persons in transition from incarceration. Each topic was addressed in a small group, allowing around thirteen students to participate in informal virtual discussions with panels of four to five community leaders, patients, or clients with lived experiences on the discussion topics. This allowed students to apply the hypothetical scenarios and national statistics taught in didactic sessions to the real-world challenges faced by New Haven residents. Seeing the community leaders in action and together with community residents prompted needed discussion of the students' own goals for their time at medical school and how they aim to integrate into these communities.

Service-based learning is a valuable tool to connect students with the surrounding community and to teach them the real-world application of lecture topics via experiential understanding [19]. The introductory course offered students optional weekend opportunities to volunteer for different New Haven community organizations. Seven organizations were featured, and 63 students participated in one or more servicebased learning opportunities. Community service locations included construction build sites, community gardens, food pantries, and soup kitchens. Notably, organizations and volunteering activities were not directly related to "medical" services, emphasizing the many different capacities in which medical students can contribute to their community.

In the past, community service was organized with fewer sites and larger rosters per site. Due to the emerged need for social distancing and limited rosters, these opportunities were expanded over two weekends and a wider array of organizations was featured. This allowed students to become familiar with smaller operations that were not previously as well-known. One student was able to introduce peers to a lesser-known organization that she had been volunteering with for the past three years that provides cooking and lifestyle 
guidance in management of chronic diseases; students contributed to the community garden that provides fresh vegetables to participants of the program.

\section{Tip 10: Incorporate Self-Reflection and Action Planning for Continued Partnerships in the Future}

An important complement to fostering a sense of belonging for medical students within the greater community involves dedicated times for self-reflection [20,21]. These periods of reflection and action setting should focus on how the student hopes to be involved in and learn from the local community throughout their medical school education. As members of this community will become the patients for whom the students care for during their clinical placements, it is imperative to understand the biases, privileges, and power structures one may participate in, and how these relate to future interactions with patients, especially from historically underserved communities as seen in New Haven [22-24].

As part of the introductory curriculum, a 90-min period was scheduled as the "Acute on Chronic Injustices SelfReflection period" where students posed two questions: (1) "why do you think we dedicated time in our curriculum to speak about structural competencies and practicing antiracism?" and (2) "write down three steps/actions that you would like to take to carry out the work started here." These questions and resulting action points are intended to serve as points to be revisited by the students, especially pertaining to how physicians can address health inequities in their immediate communities. Extending beyond this, this reflection primed students for their future involvement in the community and guided them to think more about how they can connect to New Haven beyond their roles as medical students.

Because the reflection was intended to prime students individually, the event was specifically designed for asynchronous participation where students were given independent time to reflect. However, given the modular curriculum structure at our institution, continued opportunities for reflection and follow-up were limited to curricular leaders' capacity to facilitate them. Instead, we encouraged students to independently revisit these themes.

\section{Conclusion}

The transition period to medical school and especially the first two weeks, in our experience, represent key periods for building community and a sense of belonging for incoming students with respect to their peers, their institution, and the local community surrounding their institution. In recent years, medical schools have implemented learning communities, purposefully created and highly adaptable student groups that emphasize student engagement and direct interaction between peers, faculty, and curriculum [25-27]. Individual institution reports and nationwide surveys demonstrate that learning community formats vary widely, from longitudinal advising or learning groups [28, 29] to social events [30], but with most aiming to emphasize wellbeing [25]. Regardless of a medical school's preferred learning community format, ongoing pandemic precautions have certainly hampered all efforts over the past year. Although the rapid conversion of most medical curricula to virtual formats during the COVID-19 pandemic has hampered in-person socialization, carefully considering the framework of students' peer, school, local networks have aided in the purposeful fostering of community within an introductory professional development course. Continued concerns regarding limiting unnecessary large group gatherings may require medical schools to compromise on previously fully in-person curriculum. Within our institutional experience, on-site experiences within the hospital and community centers were among the first to revert to in-person; in contrast, within-institution networking and mentorship remain largely virtual. Different medical schools may find different tips more salient depending on institutional factors (i.e., support staff capabilities, time constraints in the curriculum, and local pandemic guidelines). Beyond the ongoing concern that medical curriculum may need to be adaptable to virtual settings, these tips for building a community can be considered for all incoming students as they embark on their medical training.

Supplementary Information The online version contains supplementary material available at https://doi.org/10.1007/s40670-021-01447-z.

Acknowledgements We would like to thank all course near-peer teaching staff and faculty, administrative staff, and the coordinator for the Introduction to the Profession course, Ms. Leigh Cromey, for their invaluable contributions to the development and continuation of the course and its many components.

Author Contribution All authors designed and directed the reported curricular model. MZ and NMR wrote the manuscript. All authors provided critical feedback on the final manuscript.

Availability of Data and Material All curricular material described will be made available through the corresponding author. There is no data to be made available.

Code Availability There is no code to be made available.

\section{Declarations}

Competing Interests The authors declare no competing interests.

\section{References}

1. AAMC. 2019 Facts: Applicants and Matriculants Data. [Online]. Available: https://www.aamc.org/data-reports/students-residents/ interactive-data/2019-facts-applicants-and-matriculants-data 
2. Dunn LB, Iglewicz A, Moutier C. A conceptual model of medical student well-being: promoting resilience and preventing burnout. Acad Psychiatry. 2008;32(1):44-53. https://doi.org/10.1176/appi. ap.32.1.44.

3. Rosenthal DI, Verghese A. Meaning and the nature of physicians' work. N Engl J Med. 2016;10:375(19):1813-5. https://doi.org/10. 1056/NEJMp1609055.

4. Liu R, Carrese J, Colbert-Getz J, Geller G, Shochet R. Am I cut out for this? understanding the experience of doubt among firstyear medical students. Med Teach. 2015 Dec 2;37(12):10839. https://doi.org/10.3109/0142159X.2014.970987.

5. Weiss DF, Tilin FJ, Morgan MJ. The interprofessional health care team: Leadership and development. Jones \& Bartlett Publishers, 2014.

6. Azer SA. Challenges facing PBL tutors: 12 tips for successful group facilitation. Med Teach. 2005 Dec 1;27(8):676-81. https:// doi.org/10.1080/01421590500313001.

7. McKimm J, Ramani S, Kusurkar RA, Fornari A, Nadarajah VD, Thampy H, Filipe HP, Kachur EK, Hays R. Capturing the wisdom of the crowd: health professions' educators meet at a virtual world café. Perspectives on medical education. 2020 Dec;9(6):38590. https://doi.org/10.1007/s40037-020-00623-y.

8. Iqbal M, Velan GM, O'Sullivan AJ, Balasooriya C. Differential impact of student behaviours on group interaction and collaborative learning: medical students' and tutors' perspectives, (in eng). BMC Med Educ. 2016;16(1):217-217. https://doi.org/10.1186/ s12909-016-0730-1.

9. Jaffe LE, Lindell D, Sullivan AM, Huang GC. Clear skies ahead: optimizing the learning environment for critical thinking from a qualitative analysis of interviews with expert teachers. Perspectives on medical education. 2019 Oct;8(5):289-97. https://doi.org/ 10.1007/s40037-019-00536-5.

10. Burgess A, Mellis C. Feedback and assessment for clinical placements: achieving the right balance, (in eng). Adv Med Educ Pract. 2015;6:373-81. https://doi.org/10.2147/AMEP.S77890.

11. Siefert AL, et al. The Yale Center for Biomedical Innovation and Technology (CBIT): one model to accelerate impact from academic health care innovation, (in eng). Acad Med. 2019;94(4):528-34. https://doi.org/10.1097/acm.0000000000002542.

12. Ruzgar NM, Ahuja C, Yu KE, Sallam A, Rosenthal R, Killelea B. How We Do It: Creation of a Workforce Development-Focused Track at a Surgical Hackathon. J Surg Educ. 2020 Sep 1;77(5):102832. https://doi.org/10.1016/j.jsurg.2020.04.005.

13. Bicket M, Misra S, Wright SM, Shochet R. Medical student engagement and leadership within a new learning community. BMC Medical Education. 2010 Dec;10(1):1-6. https://doi.org/10. 1186/1472-6920-10-20.

14. Baker DM, Bhatia S, Brown S, Cambridge W, Kamarajah SK, McLean KA, Brindl N, Lapolla P, Pérez-Ajates S, Raubenheimer $\mathrm{K}, \mathrm{Xu}$ W. Medical student involvement in the COVID-19 response. The Lancet. 2020 Apr 18;395(10232):1254. https://doi.org/10.1016/ S0140-6736(20)30795-9.

15. Safarova B, Ledesma E, Luhan G, Caffey S, Giusti C. Learning from collaborative integration: the hackathon as design charrette. 2015.

16. Pathanasethpong A, Areemit R, Teerakulpisut D, Morley K, Morley $M$. Health hackathon as a venue for interprofessional education: a qualitative interview study. J Interprof Care. 2020;34(6):832-4.

17. Cardwell FS, Bisung E, Clarke AE, Elliott SJ. Original qualitative research-Hacking systemic lupus erythematosus (SLE): outcomes of the Waterlupus hackathon. Health Promotion and Chronic Disease Prevention in Canada: Research, Policy and Practice. 2020 Jul;40(7-8):235.

18. Ferreira GC, Oberstaller J, Fonseca R, Keller TE, Adapa SR, Gibbons J, Wang C, Liu X, Li C, Pham M, Dayhoff Ii GW. Iron Hack-A symposium/hackathon focused on porphyrias, Friedreich's ataxia, and other rare iron-related diseases. F1000Research. 2019;8.

19. Stewart T, Wubbena ZC. A systematic review of service-learning in medical education:1998-2012. Teach Learn Med. 2015 Apr 3;27(2):115-22. https://doi.org/10.1080/10401334.2015.1011647.

20. Mann K, Gordon J, MacLeod A. Reflection and reflective practice in health professions education: a systematic review, (in eng). Adv Health Sci Educ Theory Pract. 2009;14(4):595-621. https://doi. org/10.1007/s10459-007-9090-2.

21. Aronson L. Twelve tips for teaching reflection at all levels of medical education, (in eng). Med Teach. 2011;33(3):200-5. https://doi.org/ 10.3109/0142159x.2010.507714.

22. Hansen H, Metzl J. Structural competency in the US healthcare crisis: putting social and policy interventions into clinical practice. Bioethical Inquiry. 2016 Jun;13(2):179-83. https://doi.org/ 10.1007/s11673-016-9719-z.

23. Metzl JM, Hansen H. Structural competency: theorizing a new medical engagement with stigma and inequality, (in eng). Soc Sci Med. 2014;103:126-33. https://doi.org/10.1016/j.socscimed.2013. 06.032 .

24. Stonington SD, Holmes SM, Hansen H, Greene JA, Wailoo KA, Malina D, Morrissey S, Farmer PE, Marmot MG. Case studies in social medicine-attending to structural forces in clinical practice. N Engl J Med. 2018 Nov 15;379(20):1958-61. https://doi.org/10. 1056/NEJMms 1814262.

25. Smith S, Shochet R, Keeley M, Fleming A, Moynahan K. The growth of learning communities in undergraduate medical education, (in eng). Acad Med. 2014;89(6):928-33. https://doi.org/10. 1097/acm.0000000000000239.

26. Ferguson KJ, Wolter EM, Yarbrough DB, Carline JD, Krupat E. Defining and describing medical learning communities: results of a national survey. Acad Med. 2009 Nov 1;84(11):1549-56. [Online]. Available: https://journals.lww.com/academicmedicine/Fulltext/ 2009/11000/Defining_and_Describing_Medical_Learning.31. aspx.

27. Cruess RL, Cruess SR, Steinert Y. Medicine as a community of practice: implications for medical education, (in eng). Acad Med. 2018;93(2):185-91. https://doi.org/10.1097/acm.0000000000001826.

28. Brandl K, Mandel J, Kelly CJ. Dean's Perspective on Academic Communities at UC San Diego, School of Medicine. J Med Educ Curric Dev. 2019 May;6:2382120519848048. https://doi.org/10. $1177 / 2382120519848048$.

29. Goldstein EA, et al. Promoting fundamental clinical skills: a competency-based college approach at the University of Washington, (in eng). Acad Med. 2005;80(5):423-33. https://doi.org/10.1097/ 00001888-200505000-00003.

30. Drolet BC, Rodgers S. A comprehensive medical student wellness program-design and implementation at Vanderbilt School of Medicine, (in eng). Acad Med. 2010;85(1):103-10. https://doi.org/10.1097/ ACM.0b013e3181c46963.I.

Publisher's Note Springer Nature remains neutral with regard to jurisdictional claims in published maps and institutional affiliations. 